\title{
Expression des gènes au cours de la spermatogenèse
}

Les cellules germinales mâles subissent une différenciation importante dans le testicule, conduisant à une cellule haploïde hautement spécialisée, le spermatozoïde. A chaque stade de différenciation, $y$ compris après la méiose, correspond un mode propre d'expression de gènes. Cette grande diversité dans l'expression génique est liée à l'utilisation de modes de régulation différents : gènes spécifiquement transcrits dans les cellules germinales, promoteurs alternatifs, épissage ou polyadénylation différentiels. La signification physiologique de l'expression très spécifique de certains gènes - comme les proto-oncogènes, les gènes des protéines du choc thermique et des neuropeptides - pose des problèmes importants dont certains commencent seulement à être résolus.

\section{Robert Barouki}

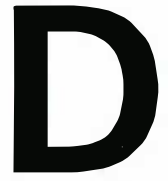

e par sa situation anatomique, ses fonctions physiologiques, et sans oublier son extraordinaire capacité à produire et exporter des cellules, le testicule est un organe à part. Bien entendu, le rôle essentiel de cet organe en endocrinologie et dans la reproduction mâle justifie les nombreux travaux qui lui sont consacrés, avec des approches physiologiques, cellulaires, biochimiques et, plus récemment, de génétique moléculaire. Sur le plan cellulaire, la fonction principale du testicule est la différenciation des cellules germinales mâles, une différenciation particulièrement originale puisqu'elle conduit à des cellules haploïdes et qu'elle est suivie d'une maturation morphologique spectaculaire. Au vu de ces propriétés, on pouvait s'attendre à une expression de gènes très spécifiques au testicule. En effet, après les premiers travaux sur les protamines et les isoenzymes de la $\mathrm{LDH}$, pour ne citer que quelques exemples, la cadence des études publiées sur l'expression de gènes spécifiques de cet organe s'est très nettement accélérée ces dernières années. Cela est dû pour une grande part à l'apport des techniques de génétique moléculaire, mais aussi à l'originalité de l'expression de très nombreux gènes dans le testicule. Même pour les gènes transcrits dans plusieurs tissus, il est fréquent de trouver un mode d'expression différent dans cet organe. Cette revue a pour objet de faire le point sur les propriétés de l'expression des gènes dans le testicule et, plus précisément, dans les cellules germinales. Il ne s'agit pas d'établir une classification exhaustive des gènes exprimés, mais plutôt d'illustrer, par quelques exemples récents, les différents modes de régulation.

\section{Rappels histologiques}

L'histologie du testicule a déjà été parfaitement décrite [1]. Ces quelques rappels pourraient aider à mieux interpréter certaines propriétés de l'expression des gènes dans cet organe. Le testicule comprend pluavenue du Maréchal-de-Lattre-de-Tassigny, 94010 Créteil, France. 


\begin{tabular}{|ll}
\hline $\begin{array}{c}\text { Spermatogonie } \\
\text { type } \mathrm{A}\end{array} \rightarrow \begin{array}{c}\text { Spermatogonie } \\
\text { type } \mathrm{B}\end{array}$ \\
Morphologie
\end{tabular}

Figure 1. Expression de quelques gènes, morphologie et ploïdie à différents stades de la spermatogenèse [1, 3, 5]. LDH : lactate déshydrogénase; PGK : phosphoglycérate kinase; hsp: heat shock protein.

sieurs types cellulaires. Les cellules de Leydig sont responsables de la synthèse d'androgènes et donc de la fonction endocrinienne de cet organe. Elles sont situées dans les espaces interstitiels entre les tubules séminifères. Ces derniers sont constitués des cellules de Sertoli et des cellules germinales. Il existe une barrière hémotesticulaire constituée principalement par les cellules de Sertoli et les jonctions étanches qu'elles établissent entre elles. Les cellules germinales sont étroitement associées aux cellules de Sertoli et leur différenciation s'effectue dans deux directions, de l'extérieur vers l'intérieur du tubule séminifère mais aussi dans le sens longitudinal, le long du tubule. Les cellules germinales mâles passent par plusieurs stades de développement (figure 1). Les cellules souches, ou spermatogonies de type $\mathrm{A}$, constituent une réserve de cellules germinales ; elles ont la capacité de se diviser mais peuvent aussi se différencier en spermatogonies de type $B$, première étape de la spermatogenèse. Après huit divisions cellulaires de spermatogonies du type $B$, la cellule entre en méiose et est alors appelée spermatocyte. Les stades précédant la première division méiotique sont souvent assez longs et sont bien caractérisés sur le plan morphologique. Les deux divisions méiotiques qui suivent sont relativement rapides et conduisent à quatre spermatides, cellules haploïdes qui vont subir une différenciation morphologique importante (la spermiogenèse) pour aboutir au spermatozoïde mûr. Au cours de cette différenciation, le spermatozoïde aura perdu une partie importante de son cytoplasme, ou corps résiduel, récupéré par les cellules de Sertoli. Il est intéressant de noter que les cellules germinales issues d'une spermatogonie primordiale maintiennent des ponts cytoplasmiques entre elles et constituent un large syncytium. Cette observation est importante concernant l'expression des gènes, surtout s'il est prouvé que les ponts permettent le passage de macromolécules. Dans ce cas, une possible hétérogénéité dans l'expression d'un gène dans les cellules germinales pourrait ne pas se refléter dans le contenu cellulaire en protéine correspondante.

\section{Systèmes expérimentaux}

Les rongeurs constituent les modèles expérimentaux les plus utilisés pour étudier la spermatogenèse [2]. La souris présente, en particulier, de nombreux avantages:

- il existe plusieurs mutants stériles connus caractérisés par un arrêt de la spermatogenèse à un stade bien précis ;

- l'apparition des différents types de cellules germinales se fait de manière séquentielle après la naissance et avec une cinétique relativement précise. Selon l'âge de l'animal, les testicules sont plus ou moins riches en un type donné de cellules germinales;

- il est relativement facile de purifier des cellules germinales à un stade donné, en particulier par des techniques de centrifugation ;

- l'impossibilité de réaliser des cultures de cellules germinales rend le modèle animal encore plus attractif. En particulier, les souris transgéni- 


\section{RÉFÉRENCES}

1. Clermont Y. Spermatogenèse et épithélium séminifère. In : Schaison G, Bouchard P, Mahoudeau J, Labrie F, eds. Médecine de la reproduction masculine. Paris : Flammarion, 1984: 40-50

2. Propst F, Rosenberg M, Van de Woude G. Proto-oncogene expression in germ cell development. Trends Genet 1988 ; 4 : 183-7.

3. Erickson R. Post-meiotic gene expression. Trends Genet 1990 ; 6 : 264-9.

4. Hecht NB. The molecular biology of spermatogenesis : regulation of mammalian protamines from gene to protein. In : Serio $\mathrm{M}$, ed. Perspectives in Andrology. New York : Raven Press 1989 : 25-35.

5. Willison K, Ashworth A. Mammalian spermatogenic gene expression. Trends Genet $1987 ; 3: 351$.

6. Wolgemuth D, Watrin F. List of cloned mouse genes with unique expression patterns during spermatogenesis. Mammalian Genome $1991 ; 1: 283-8$

7. Markert CL, Shakler JB, Whitt GS. Evolution of a gene: multiple genes for LDH isozymes provide a model of the evolution of gene structure, function and regulation. Science $1975 ; 189$ : 102-14.

8. McCarrey JR, Thomas K. Human testisspecific PGK gene lacks introns and possesses characteristics of a processed gene. Nature $1987 ; 326: 501-4$.

9. Taira M, Iisaza T, Shimada H, Kudoh J, Shimizu N, Tatibana M. A human testisspecific mRNA for phosphoribosylpyrophosphate synthetase that initiates from a nonAUG codon. J Biol Chem 1990; 265 : 16491-7.

10. Robertson N, Pomponio R, Mutter G, Morton C. Testis-specific expression of the human MYCL2 gene. Nucleic Acids Res $1991 ; 19: 3129-37$

11. Ashworth A, Skene B, Swift S, LovellBadge R. Z $f a$ is an expressed retroposon derived from an alternative transcript of the ques ont permis d'entamer l'analyse des sites responsables de la spécificité cellulaire d'expression de certains promoteurs et ceux permettant la régulation traductionnelle de l'expression de protéines du testicule.

Si l'essentiel des travaux expérimentaux a eu lieu chez la souris et le rat, l'étude de la spermatogenèse chez l'homme pourra profiter des progrès techniques importants qui ont pu être réalisés récemment, permettant notamment, de séparer les différents types de cellules germinales humaines (B. Jegou, communication personnelle). De plus, les séminomes peuvent constituer, dans une certaine mesure, des modèles in vitro pour l'étude des cellules germinales.

\section{$Y$ a-t-il expression de gènes après la méiose ?}

Bien que cette question ait été l'objet de controverses, plusieurs études ont récemment montré qu'il y avait bien transcription spécifique de certains gènes après la méiose. En effet, la comparaison de produits de traduction in vitro d'ARN messagers, provenant de spermatides et de spermatocytes, a montré une différence très nette dans la nature des protéines synthétisées [3]. Une étude par électrophorèse sur gel en deux dimensions montre qu'il existe en réalité plus de protéines différentes que de protéines communes. Ces différences sont probablement encore plus importantes si l'on considère toutes les protéines non révélées par cette technique. Ces travaux suggèrent fortement l'existence d'une transcription post-méiotique spécifique mais, comme nous le verrons plus loin, ne constituent pas une preuve directe de cette hypothèse.

Un des exemples les plus frappants de modification post-méiotique est le changement de la composition en protéines de la chromatine, entraînant des modifications bien connues de la texture chromatinienne au cours de la spermatogenèse. En effet, dès le début de la méiose, des histones spécifiques du testicule remplacent progressivement les histones somatiques [4]. Après la méiose, ces histones sont elles-mêmes remplacées par d'autres protéines basiques, les pro- téines de transition qui, à leur tour, vont céder la place aux protamines. Les messagers des protamines et des protéines de transition sont détectés uniquement dans les cellules haploïdes. Pour certaines de ces protéines, par exemple l'histone $2 \mathrm{~B}$, la régulation du taux du messager au cours du développement a été démontrée par des expériences de Northern blot mais également par des expériences de transcription sur noyaux isolés [3]. Des résultats analogues ont été obtenus pour une autre catégorie de gènes codant pour des enzymes métaboliques comme la LDH-X.

L'ensemble de ces observations démontre bien qu'il existe une activité transcriptionnelle dans les cellules germinales, avant, pendant et après la méiose. A chaque stade de différenciation correspond une expression très spécifique de gènes [3] ; le cas des protéines chromatiniennes cité plus haut est une bonne illustration de la cascade d'événements transcriptionnels accompagnant la spermatogenèse. Cette notion doit être tempérée par plusieurs observations : (1) il existe des gènes qui sont exprimés tout au long de la spermatogenèse, comme le montre l'analyse par gel en deux dimensions et l'étude spécifique de certains gènes; (2) à la régulation transcriptionnelle vient se greffer une régulation de type traductionnel que nous reverrons plus loin. En effet, des ARN peuvent être synthétisés et stockés dans le cytoplasme à un stade donné puis traduits en protéines spécifiquement à un stade ultérieur. Enfin, si la transcription a bien lieu après la méiose, elle est interrompue au cours de la spermiogenèse à un moment où les modifications chromatiniennes deviennent trop importantes.

\section{Propriétés générales de l'expression des gènes dans le testicule}

Un examen, même rapide, de la littérature montre que l'expression des gènes dans le testicule est différente de celle des autres tissus. Bien sûr, ce type d'analyse est biaisé parce que bien souvent seules les différences sont publiées. Néanmoins, l'accumulation de données est telle que l'on est en droit de penser que cet organe 
est un système privilégié pour étudier une expression alternative de gènes. Il est vrai que dans de nombreux cas l'expression des gènes a été étudiée sur l'organe entier et l'origine cellulaire n'a pas été déterminée. Dans cette revue, seuls les gènes exprimés dans les cellules germinales seront analysés. Dans une revue publiée en 1987, Willison et Ashworth [5] ont réparti les gènes exprimés de manière spécifique au cours de la spermatogenèse en trois grandes classes : (1) Les gènes exprimés exclusivement durant la spermatogenèse ; (2) les gènes codant pour une isozyme ou un isotype spécifique du testicule alors que la fonction codée est aussi exprimée dans les cellules somatiques ; (3) les gènes dont l'expression est modifiée dans les cellules germinales mâles. Cette modification peut être soit qualitative (taille du messager), soit quantitative. Cette classification est actuellement acceptée et permet de distinguer différents types de modifications.

- Gènes codant pour des fonctions spécifiques

Les gènes représentatifs de la première classe sont ceux des protamines et des protéines de transition dont la fonction est spécifiquement associée à la spermatogenèse [4]. D'autres gènes, comme celui de la préproacrosine, codent pour une protéine spécifique de la lignée germinale mâle. Il existe aussi des gènes dont la fonction n'est pas connue mais dont l'expression se restreint à une ou plusieurs étapes de la spermatogenèse [6]. Il s'agit, par exemple, de certains gènes localisés au niveau du complexe $\mathrm{t}$ sur le chromosome 17. La présence de gènes très spécifiques de la spermatogenèse ne constitue pas une situation particulièrement originale puisque, dans chaque tissu, on retrouve ce type d'expression spécifique.

- Gènes codant pour des isoenzymes ou isotypes spécifiques

L'expression d'isoenzymes spécifiques du testicule est un phénomène connu depuis les années 1950 ; l'exemple classique est la lactate déshydrogénase $\mathrm{X}$ (LDH-X ou LDH-C), qui est spécifique de la lignée germinale mâle, contrairement à la $\mathrm{LDH}-\mathrm{A}$ et à la LDH-B [7]. Un autre exemple très étudié est celui de la PGK (phosphoglycérate kinase), enzyme de la glycolyse, qui existe sous deux formes $\mathrm{m} / \mathrm{s} n^{\circ} 6$, vol. 8 , juin-juillet 92 isoenzymatiques, $\mathrm{PGK}_{1}$ et $\mathrm{PGK}_{2}[8]$. L'étude du système $P G_{1}-P_{2} K_{2}$ est instructive à plus d'un titre. La $\mathrm{PGK}_{1}$ est l'isozyme somatique dont le gène est localisé sur le chromosome $\mathrm{X}$. La $\mathrm{PGK}_{2}$ possède un gène autosomique et n'est exprimé qu'au cours de la spermatogenèse dans les spermatocytes et les spermatides. La comparaison des deux gènes est intéressante sur le plan de la diversification génique [8]. En effet, le gène de la $\mathrm{PGK}_{1}$ comprend dix introns alors que le gène de la $\mathrm{PGK}_{2}$ est sans introns, possède une séquence de type poly $\mathrm{A}$ et est bordé par des séquences répétées. Ces propriétés sont celles des " rétroposons ", dont la structure rappelle celle de nombreux pseudogènes. Le fait que le gène de $\mathrm{PGK}_{2}$, avec ce type de structure, soit malgré tout fonctionnel pourrait résulter d'une forte pression de sélection en raison du rôle clé de cette enzyme dans le métabolisme du fructose. Or le chromosome $\mathrm{X}$ (et donc le gène $\mathrm{PGK}_{1}$ ) est très largement inactivé au cours de la spermatogenèse, en particulier dans les cellules haploïdes. D'où la nécessité d'exprimer la PGK à partir d'un gène somatique.

Le cas de la PGK est similaire à celui de la phosphoribosylpyrophosphate synthétase [9]. Plusieurs isoenzymes PRPS existent : deux sont somatiques et leurs gènes sont sur le chromosome $\mathrm{X}$; une est spécifique du testicule et son gène est autosomique. Cette règle n'est cependant pas absolue. En effet, $M y c L_{2}$, qui est apparenté au produit du proto-oncogène $c$ $m y c$, est exprimé dans le testicule à partir d'un gène localisé sur le X [10]. Sachant que ce chromosome est inactivé, on peut dans ce cas invoquer soit une inactivation incomplète du $\mathrm{X}$, soit une dissociation possible entre transcription et traduction, l'ARN étant synthétisé avant l'inactivation du X et traduit ultérieurement.

Le gène codant pour $M y c L_{2}$ partage avec celui de $\mathrm{PGK}_{2}$ une autre propriété, l'absence d'introns. D'autres gènes exprimés dans le testicule adulte sont aussi dépourvus d'introns, comme par exemple le gène d'une sous-unité de la pyruvate déshydrogénase 2 chez l'homme, et celui de la protamine du poulet [10]. La caractérisation d'autres gènes permettra de savoir dans quelle mesure l'expression de ce type de gènes est caractéristique de la spermatogenèse.

Récemment, l'expression de gènes localisés sur le chromosome Y a été étudiée dans le testicule. La famille des gènes $Z$ fy code pour une protéine à doigts à zinc. Chez la souris, deux de ces gènes sont sur le chromosome $\mathrm{Y}$ et sont exprimés dans le testicule et dans d'autres tissus. Cependant, un troisième membre de cette famille est, lui, autosomique, et son expression est spécifique du testicule. Ce gène a aussi une structure rappelant celle des rétroposons [11].

\section{Expression alternative d'un gène}

Dans ce cas de figure, très courant, un même gène est transcrit de manière différente entre les cellules germinales et les cellules somatiques. Bien sûr, une telle variabilité peut se retrouver pour chaque tissu de manière spécifique. Cependant, la grande fréquence de cette variabilité distingue le testicule. Les différents modes de régulation connus sont utilisés et sont illustrés sur la figure 2, p. 536.

- Promoteurs différents

On retrouve pour certains gènes, deux promoteurs différents: l'un est exprimé dans les cellules somatiques et l'autre dans les cellules germinales. L'exemple le plus frappant est celui de l'enzyme de conversion de l'angiotensine. Cette enzyme est constituée de deux domaines semblables probablement issus d'une duplication de gène. Dans le testicule, la protéine n'est constituée que d'un seul des deux domaines et rappelle par sa structure la protéine ancestrale [12]. Le gène est constitué de 26 exons exprimés dans les cellules somatiques. Or, dans le $12^{\mathrm{e}}$ intron du gène, un promoteur d'expression spécifiquement testiculaire a été mis en évidence, entraînant la synthèse d'une protéine beaucoup plus courte [13]. Ainsi, dans ce cas, l'initiation alternative de la transcription entraîne la synthèse de deux protéines très différentes mais dont la fonction est probablement similaire. Deux initiations différentes peuvent cependant entraîner la synthèse de deux ARNm codant pour des protéines dont la fonction est différente. Ainsi, la calspermine, qui est spécifique des cellules germinales, est issue 
Groupe I : Gènes uniquement exprimés dans le testicule

\section{RÉFÉRENCES}

12. Lattion A, Soubrier F, Allegrini J, Hubert C, Corvol P, Alhenc-Gelas F. The testicular transcript of the angiotensin Iconverting enzyme encodes for the ancestral, non-duplicated form of the enzyme. Febs Lett 1989 ; 252 : 99-104.

13. Howard T, Shai S, Langford K, Martin B, Bernstein K. Transcription of testicular angiotensin-converting enzyme (ACE) is initiated within the 12th intron of the somatic ACE gene. Mol Cell Biol 1990; 10 : 4294-302.

14. Means A, Cruzalegui F, LeMagueresse B, Needleman D, Slaughter G, Ono T. A novel $\mathrm{Ca}^{2}+/$ calmodulin-dependent protein kinase and a male germ cell-specific calmodulin-binding protein are derived from the same gene. Mol Cell Biol 1991; 11 : 3960-71.

15. Teruya J, Kutsunai S, Spear DH, Edwards P, Clarke C. Testis-specific transcription initiation sites of rat farnesyl pyrophosphate synthetase mRNA. Mol Cell Biol $1990 ; 10: 2315-26$

16. Kilpatrick DL, Zinn SA, Fitzgerald M, Higuchi H, Sabol S, Meyerhardt J. Transcription of the rat and mouse proenkephalin genes is initiated at distinct sites in spermatogenic and somatic cells. Mol Cell Biol 1990 ; $10: 3717-26$

17. Villasante A, Wang D, Dobner P, Dolph P, Lewis S, Cowan N. Six mouse $\alpha$-tubulin mRNAs encode five distinct isotypes : testisspecific expression of two sister genes. Mol Cell Biol 1986 ; 6 : 2409-19.

18. Waeber G, Meyer T, Le Sieur M, Hermann H, Gérard N, Habener J. Developmental stage-specific expression of cyclic adenosine 3', 5'-monophosphate response element-binding protein CREB during spermatogenesis involves alternative exon splicing. Mol Endocrinol 1991; 5 : 1418-30.

19. Foulkes NS, Mellström B, Benusiglio E, Sassone-Corsi P. Developmental switch of CREM function during spermatogenesis : from antagonist to activator. Nature 1992 ; $355: 80-84$

20. Haugen T, Paulssen E, Gordeladze J, Hansson V. A unique mRNA species for the $\alpha$ subunit of $\mathrm{G}_{\mathrm{s}}$ is present in rat haploïd germ cells. Biochem Biophys Res Commun 1990 ; $168: 91-8$.

21. Meijer S, Hermans A, von Lindern M, et al. Molecular characterization of the testis specific $c-a b l$ mRNA in mouse. EMBO J $1987 ; 6: 4041-8$.

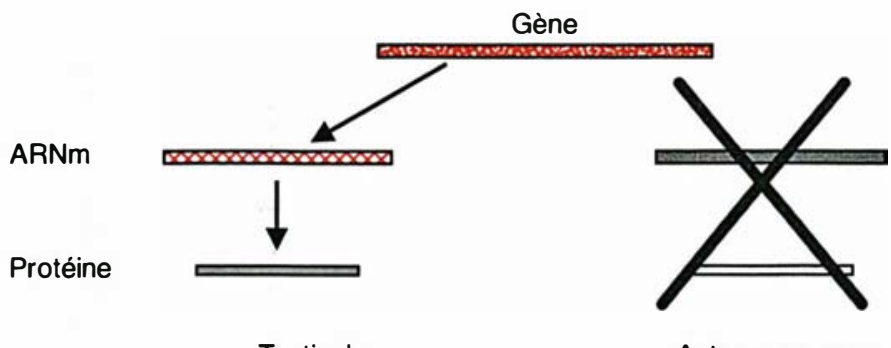

Testicule

Autres organes

ex. : Protamine

Protéine de transition

Groupe II : Isoformes spécifiques à partir de gènes différents

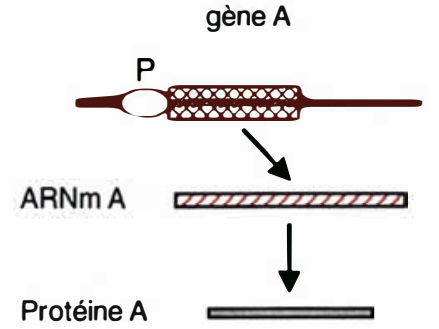

Testicule

ex. : Phosphoglycérate kinase 2 Autosome

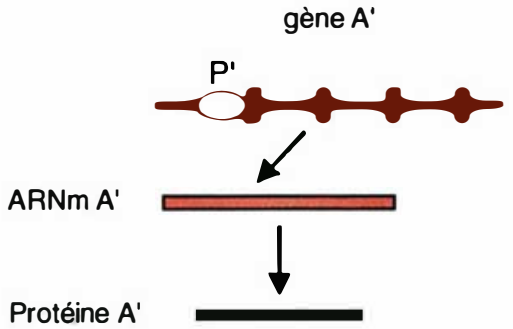

Autres organes

ex. : Phosphoglycérate kinase 1 Chromosome $X$

Figure 2. Différents modes d'expression des gènes dans les cellules germinales mâles. Les trois groupes décrits par Willison et Ashworth [5] sont illustrés par des exemples. Le groupe III englobe différents modes de régulation. Dans l'exemple choisi, l'utilisation d'un promoteur différent entraîne la synthèse d'une protéine différente. De même, l'épissage différentiel de pré-ARN CREB dans le testicule introduit un codon de terminaison de la traduction et donc conduit à la synthèse d'une protéine tronquée. Dans le cas du gène c-abl, les ARN diffèrent par leur partie $3^{\prime}$ non codante entre le testicule et les autres tissus, du fait de l'utilisation de signaux alternatifs de polyadénylation (AATAAA). Les protéines sont cependant identiques.

de la même unité génétique qu'une protéine kinase dépendante de la calmoduline [14]. Il existe un autre cas de figure illustré par le gène de la farnésyl pyrophosphate synthétase (FPS). Le site d'initiation spécifique des cellules germinales se situe en amont du site somatique [15]. L'ARNm ainsi synthétisé comprend une séquence 5' non traduite plus longue qui entraîne une réduction importante de l'efficacité de la traduction. Ainsi, la modification du site d'initiation de la transcription altère la capacité de traduction du messager, alors que la protéine synthétisée est identique. D'autres gènes voient aussi leurs sites d'initiation modifiés dans les cellules germinales, comme par exemple la proenképhaline et la proopiomélanocortine [16]. Pour ce qui est de l' $\alpha$ tubuline, un site d'initiation différent va entraîner la synthèse d'une protéine tronquée par l'absence de méthionine initiatrice [17].

\section{- Épissage alternatif}

Ce mode de régulation est aussi retrouvé dans le testicule. Parmi les quelques gènes étudiés, ceux des protéines CREB et CREM offrent un intérêt particulier sur le plan des régulations géniques. La protéine CREB, 


\section{Groupe III : Expression alternative d'un même gène}

Promoteurs différents

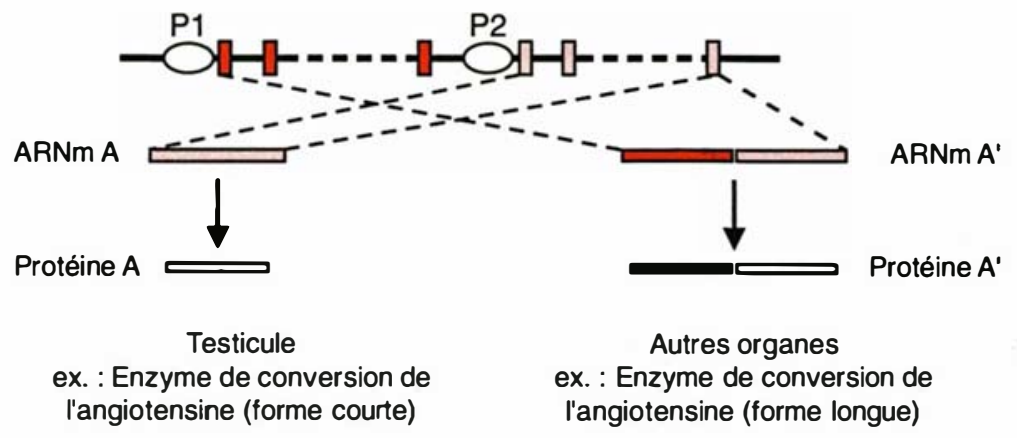

Épissage différentiel

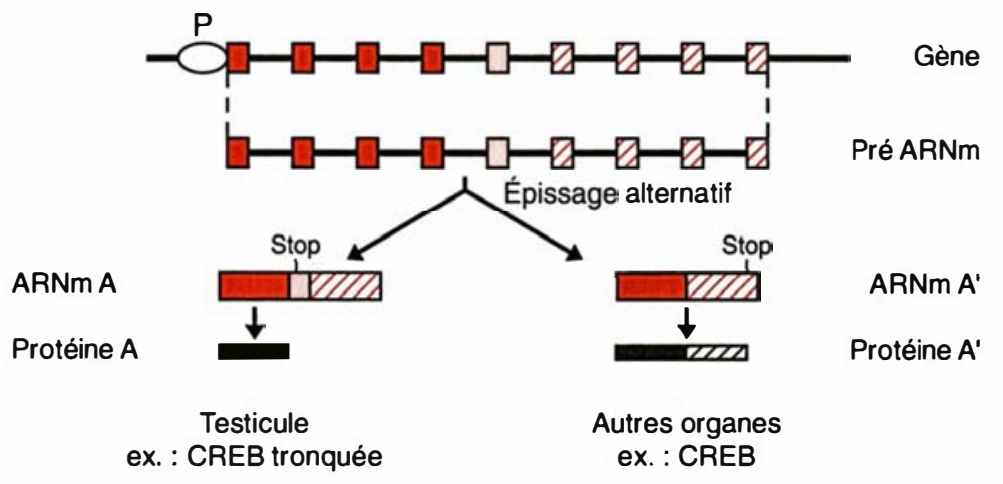

Polyadénylation différente

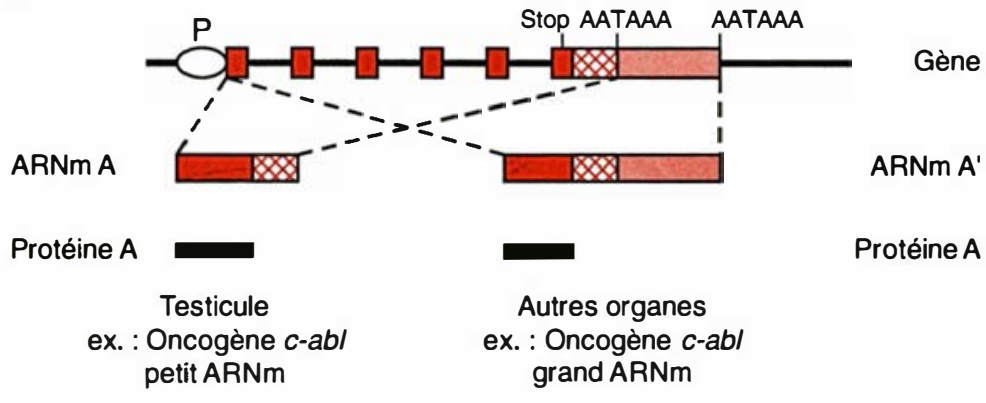

qui est un facteur transcriptionnel de la famille des leucine zippers, est le médiateur de certains effets transcriptionnels de l'AMPc. Dans les cellules germinales, un épissage alternatif du pré-ARNm conduit à la synthèse d'une protéine tronquée ayant perdu le site de liaison à l'ADN et le site de translocation nucléaire [18]. Le rôle exact de cette protéine tronquée n'est pas connu ; de par sa structure et sa $m / s n^{\circ} 6$, vol. 8 , juin-juillet 92 dépourvues du domaine d'activation de la transcription sont retrouvées dans quelques tissus et, en s'associant à CREB, pourraient constituer des inhibiteurs des effets transcriptionnels de cette protéine. Dans les cellules germinales mâles, une forme résultant d'un épissage différent est très fortement et spécifiquement exprimée. Cette forme comprend des domaines d'activation de la transcription, et pourrait constituer un activateur de la transcription sensible à l'AMPc [19]. Le rôle de cette forme de la protéine CREM pourrait être d'induire certains gènes au cours de la spermatogenèse.

Il est intéressant de noter que d'autres protéines impliquées dans la production ou le mécanisme d'action de l'AMPc ont une expression différente dans les cellules germinales. Citons par exemple la sous-unité $\alpha$ de la protéine $\mathrm{Gs}$ qui est codée par un ARNm spécifique de ces cellules [20]. Cet ARNm est trop petit pour pouvoir coder pour une protéine fonctionnelle normale. L'épissage alternatif, ainsi que l'initiation différente, pourraient constituer des moyens économiques d'adaptation de l'expression des gènes à une structure et un environnement cellulaire différents.

- Polyadénylation alternative

La taille des messagers de certains gènes peut varier d'un tissu à l'autre en raison d'un choix différent du site de polyadénylation. Cela se traduit par une taille différente de la séquence 3 ' non codante avec, potentiellement, une régulation différente de la traduction ou de la stabilité des messagers. Plusieurs cas ont été rapportés où la polyadénylation est différente dans le testicule. Le protooncogène $c-a b l$ est ainsi exprimé dans les cellules germinales sous forme de messagers de tailles inférieures par rapport à ceux des cellules somatiques [21]. Le signal de polyadénylation reconnu est un signal inhabituel différent du consensus. On ne sait pas encore si cette propriété est spécifique du testicule. Cependant, l'analyse d'autres messagers présentant une polyadénylation alternative $\left(\beta_{1}\right.$-galactosyl transférase, par exemple) [22] permet de penser que, dans cet organe, c'est le site de polyadénylation proximal qui est préférentiellement choisi, conduisant à des ARNm plus petits. Il est possible que la 
machinerie de polyadénylation ou de terminaison de la transcription soit différente dans le testicule et favorise les sites proximaux. Concernant les autres modes de régulation (initiation, épissage), il ne semble pas exister de règle générale quant à la taille du messager testiculaire.

\section{Adaptation physiologique}

\section{RÉFÉRENCES}

22. Shaper N, Wright W, Shaper J. Murine $\beta 1,4$-galactosyltransferase : both the amounts and structure of the mRNA are regulated during spermatogenesis. Proc Natl Acad Sci USA $1990 ; 87: 791-5$.

23. Bunick D, Johnson $P$, Johnson $T$, Hecht N. Transcription of the testis-specific mouse protamine 2 gene in a homologous in vitro transcription system. Proc Natl Acad Sci USA $1990 ; 87: 891-5$.

24. Robinson M, McCarrey J, Simon M. Transcriptional regulatory regions of testis-specific $\mathrm{PGK}_{2}$ defined in transgenic mice. Proc Natl Acad Sci USA 1989; 86 : 8437-41.

25. Langford K, Shai S, Howard T, Kovac M, Overbeck P, Bernstein K. Transgenic mice demonstrate a testis-specific promoter for angiotensin-converting enzyme. $J$ Biol Chem 1991 ; 266 : 15559-62.

26. Goto M, Tamura T, Mikoshiba K, Masamune Y, Nakanishi Y. Transcription inhibition of the somatic-type phosphoglycerate kinase 1 gene in vitro by a testis-specific factor that recognizes a sequence similar to the binding site for Ets oncoproteins. Nucleic Acids Res 1991 ; 19 : 3959-63.

27. Ariel M, McCarrey J, Cedar H. Methylation patterns of testis-specific genes. Proc Natl Acad Sci USA 1991 ; 88 : 2317-21.

28. Choi Y, Chae C. DNA hypomethylation and germ cell-specific expression of testisspecific H2B histone gene. J Biol Chem 1991 ; 266 : 20504-11.

29. Jordan BR. Ilots HTF, le gène annoncé. médecine/sciences $1991 ; 7$ : 153-60.

30. Chaillet J, Vogt T, Beier D, Leder P. Parental-specific methylation of an imprinted transgene is established during gametogenesis and progressively changes during embryogenesis. Cell 1991; 66 : 77-83.

31. Babinet C. L'empreinte génomique parentale. médecine/sciences $1992 ; 8$ : 65-70.

32. Kwon Y, Hecht N. Cytoplasmic protein binding to highly conserved sequences in the 3' untranslated region of mouse protamine 2 mRNA, a translationally regulated transcript of male germ cells. Proc Natl Acad Sci
Devant tant de changements dans l'expression génique, on est tenté de rechercher les bases physiologiques ayant entraîné la sélection et le maintien de ce type d'expression. Nous avons déjà mentionné le rôle des protamines, des protéines de transition et des histones spécifiques dans les modifications chromatiniennes des cellules germinales. De même, les modifications morphologiques importantes au cours de la spermiogenèse peuvent nécessiter la sélection de nouveaux types de protéines du cytosquelette comme l'actine et la tubuline. Une autre caractéristique du testicule est la température optimale, qui est de quelques degrés inférieure à celle du reste du corps. De nouvelles isozymes pourraient être mieux adaptées à ce type de conditions. Par ailleurs, le métabolisme des spermatozoïdes est différent de celui d'autres cellules y compris des cellules germinales immatures. En particulier, l'utilisation de fructose et de lactate y est privilégiée par rapport à celle du glucose, ce qui pourrait correspondre à l'expression de nouvelles isozymes de la glycolyse. D'une manière plus générale, et comme nous le verrons un peu plus loin, l'ADN des cellules germinales subit des modifications comme la méthylation. Ces modifications pourraient inactiver des régions de certains chromosomes nécessitant l'activation d'autres gènes codant pour des isozymes. Ces modifications d'ADN pourraient non seulement avoir un rôle dans l'expression des gènes des cellules germinales, mais aussi être importants pour les étapes ultérieures comme la fécondation et l'embryogenèse.

\section{Modes de régulation}

Les gènes exprimés dans le testicule sont contrôlés à la fois sur le plan transcriptionnel et sur le plan traductionnel. L'étude des régulations trans- criptionnelles a été retardée en raison de l'absence de système cellulaire simple permettant le dosage de l'activité promotrice et l'analyse de sa spécificité. Trois approches ont cependant pu être utilisées. En premier lieu, le clonage des promoteurs spécifiques a été entrepris et la liaison de facteurs transcriptionnels a été déterminée. Une analyse fonctionnelle a été entreprise grâce aux techniques de transcription in vitro en présence d'extraits nucléaires de tissus différents [23]. Ainsi, des éléments positifs et négatifs ont pu être mis en évidence dans le promoteur de la protamine 1 [4]. Enfin, certains promoteurs ont pu être analysés grâce aux souris transgéniques. De la sorte, la spécificité cellulaire et la spécificité d'expression liée au stade de différenciation ont pu être vérifiées pour des fragments de promoteurs spécifiques tels que ceux des gènes de la protamine 1 , de la $\mathrm{PGK}_{2}[24]$ et de l'enzyme de conversion [25]. Il n'est pas encore possible d'attribuer à une séquence particulière un rôle déterminant dans la spécificité d'expression.

En fait, beaucoup de ces promoteurs contiennent, de façon banale, une boîte TATA et/ou une ou plusieurs séquences riches en $\mathrm{GC}$, comme de très nombreux gènes non exprimés dans les testicules. On ne peut, en réalité, exclure l'hypothèse d'une expression par défaut, l'absence d'un élément négatif permettant l'expression dans les cellules germinales. Un tel élément vient d'être mis en évidence dans le promoteur de la $\mathrm{PGK}_{1}$ dont l'expression s'éteint aux premiers stades de la méiose [26].

La méthylation de l'ADN joue un rôle important dans l'expression des gènes. Même s'il est difficile de généraliser, il est admis que la déméthylation de certains sites d'ADN est associée à une activation de gènes dans les tissus où ils sont exprimés. Le cas des cellules germinales est cependant tout à fait particulier. En effet, si un gène exprimé spécifiquement dans ces cellules est déméthylé, ce processus doit être réversible, soit à la fin de la spermatogenèse, soit au cours de l'embryogenèse pour maintenir la même spécificité d'expression dans la génération suivante. C'est en effet le cas de la $\mathrm{PGK}_{2}$, dont le gène est déméthylé dès le stade sper- 
matogonie puis reméthylé dans le spermatozoïde [27]. Il en est de même pour le gène de l'histone $2 \mathrm{~B}$ spécifique du testicule, mais, dans ce cas, la méthylation a lieu au cours de l'embryogenèse [28]. Cependant, la plupart des gènes spécifiques du testicule sont précédés de ce que l'on appelle les îlots $\mathrm{CpG}$ (séquences riches en dinucléotide CG [29] ; or ces îlots ne sont méthylés ni dans les cellules germinales ni dans les autres cellules de l'organisme [27]. En réalité, ces îlots sont retrouvés essentiellement en amont des gènes de fonction ubiquitaire, mais aussi en amont de quelques gènes à expression spécifique de tissu. Dans ce cas, la méthylation ne joue pas de rôle direct dans la spécificité d'expression qui est déterminée par l'intervention directe de facteurs transcriptionnels spécifiques de tissu, activateurs ou inhibiteurs.

La méthylation est aussi impliquée dans le phénomène d'empreinte parentale par lequel un gène peut s'exprimer différemment s'il provient du père ou de la mère. Cette empreinte doit nécessairement être modifiée à chaque génération. Cette modification de la méthylation se fait dans les cellules germinales primordiales. Une méthylation différente a lieu pendant la spermatogenèse et le type paternel n'apparaît qu'après fécondation, au cours de l'embryogenèse [ 30 , 31].

\section{Régulations traductionnelles}

Des régulations au niveau traductionnel ont été révélées par la dissociation entre l'apparition d'un ARNm et de la protéine pour laquelle il code au cours de la spermatogenèse. A titre d'exemple, les ARNm des protamines de souris sont synthétisés dans les spermatides avant l'arrêt de la transcription. Or les protéines n'apparaissent que trois à sept jours plus tard. Entretemps, les ARNm sont stockés sous forme de ribonucléoprotéines, avant d'être associés aux polysomes et d'être traduits [4]. Des expériences utilisant les souris transgéniques avec des messagers chimériques, hormone de croissance/protamine, ont permis de montrer que cette régulation est liée à la partie 3' non codante du messager de la protamine 2 . Récemment, une pro$\mathrm{m} / \mathrm{s} n^{\circ} 6$, vol. 8, juin-juillet 92 téine se liant à une séquence présente dans la partie 3' non codante du messager de la protamine a été mise en évidence [32] ; cette protéine pourrait être responsable de la régulation traductionnelle. Par ailleurs, lors du passage des messagers de la protamine dans la fraction polysomale, la taille de la queue poly-A diminue considérablement. Ce phénomène ne semble cependant pas général et ne s'applique par exemple pas à la LDH-X.

\section{Spermatogenèse et oncogènes}

Parmi les gènes exprimés dans les cellules germinales, on trouve de nombreux proto-oncogènes [2]. Cette expression est assez surprenante dans la mesure où ces gènes sont souvent impliqués dans la croissance cellulaire : or, on retrouve les produits de certains proto-oncogènes comme $c$-mos, $c$-int et $c$-abl dans les cellules haploïdes, donc après la méiose. La fonction des produits de ces gènes dans la spermatogenèse est assez mal connue, d'autant plus que certains sont exprimés sous une forme modifiée (protéine tronquée). On ne peut exclure que ces protéines soient impliquées dans la phase initiale de l'embryogenèse. Le proto-oncogène $c$-mos est un cas particulier puisque l'on sait qu'il est aussi exprimé dans les ovules et qu'il correspondrait au facteur cytostatique ([33], $m / s n^{\circ} 2$, vol. 6, p. 162), notion d'ailleurs contestée par des résultats récents [34]. Il pourrait donc peut-être jouer un rôle particulier au cours de la méiose.

\section{Gènes exprimés au cours de l'embryogenèse}

Une autre constatation que l'on peut faire à l'examen des gènes exprimés au cours de la spermatogenèse est que certains sont aussi exprimés au cours de l'embryogenèse [6]. Il s'agit par exemple de gènes homéotiques Hox 1.4 et de certains proto-oncogènes [35]. Il en est de même pour le gène $Z f y$, qui code pour une protéine de liaison à l'ADN contenant des doigts à zinc [6]. Le rôle exact de ces protéines au cours de la spermatogenèse reste à déterminer. De plus, il n'est souvent pas démontré que ces protéines sont effectivement synthétisées dans les cellules germinales. Il n'en demeure pas moins que ces régulations communes à l'embryogenèse et à la spermatogenèse méritent d'être étudiées sur les plans moléculaire et physiologique.

\section{Gènes exprimés dans le système nerveux}

Plusieurs gènes exprimés dans le système nerveux le sont aussi dans les cellules germinales mâles. Il s'agit, par exemple, de précurseurs de neuropeptides comme la proopiomélanocortine et la proenképhaline [6] mais aussi des enzymes de biosynthèse de neuropeptides et de neurotransmetteurs comme l'endopeptidase Ep24.15 [36] et la glutamate décarboxylase [37]. Il existe même des isozymes spécifiquement exprimées à la fois dans le testicule et le cerveau, comme par exemple une des formes de la glutathion $\mathrm{S}$ transférase [38]. De plus, parmi les facteurs de croissance exprimés dans les cellules germinales, on trouve le TGF $\beta$ mais également le NGF [39]. D'ailleurs le testicule contient des récepteurs NGF de basse affinité. Ces facteurs de croissance pourraient jouer un rôle dans le développement et la maturation des cellules germinales mâles. Quant aux neuropeptides, leur rôle testiculaire n'est pas connu. Une étude récente vient illustrer de manière frappante l'analogie partielle entre les expressions de gène dans le testicule et dans le système nerveux. En effet, parmi des dizaines de récepteurs olfactifs exprimés dans le tissu olfactif, certains sont aussi exprimés dans les cellules germinales mâles et pourraient jouer un rôle dans le chimiotactisme des spermatozoïdes au cours de la fécondation ([40], $m / s \quad n^{\circ} 3$, vol. 8 , p. 293).

\section{Protéines de choc thermique}

Des protéines de choc thermique sont exprimées dans les cellules germinales mâles. Les plus étudiées sont celles de la famille hsp70. Certains gènes de cette famille sont spécifiquement exprimés à des stades déterminés de la spermatogenèse, soit avant la première division méiotique, soit après la 


\section{RÉFÉRENCES}

33. Sagata N, Watanabe N, Van de Woude $\mathrm{G}$, Ikawa $\mathrm{Y}$. The $c$-mos proto-oncogene product is a cytostatic factor responsible for meiotic arrest in vertebrate eggs. Nature 1989 ; $342: 512$.

34. Yew N, Mellini NL, Van Woude GF. Meiotic initiation by the Mos protein in Xenopus. Nature 1992 ; 355 : 649-52.

35. Wolgemuth D, Viviano C, GizangGinsberg E, Frohman M, Joyne A, Martin $G$. Differential expression of the mouse homeobox-containing gene Hox-1.4 during males germ cell differentiation and embryonic development. Proc Natl Acad Sci USA $1987 ; 84$ : 5813-7.

36. Orlowski M, Reznik S, Ayala J, Pierotti A. Endopeptidase 24.15 from rat testes. Biochem J 1989 ; 261 : 951-8.

37. Persson H, Pelto-Huikko M, Metsis $\mathbf{M}$, et al. Expression of the neurotransmittersyntheşizing enzyme glutamic acid decarboxylase in male germ cells. Mol Cell Biol $1990 ; 10: 4701-11$

38. Campbell E, Takahashi Y, Abramovitz M, Peretz M, Listowsky I. A distinct human testis and brain $\mu$-class glutathione $\mathrm{S}$ transferase. J Biol Chem 1990 ; 265 : 9188-93.

39. Ayer-Lelievre C, Olson L, Ebendal T, Hallböök F, Persson $H$. Nerve growth factor mRNA and protein in the testis and epididymis of mouse and rat. Proc Natl Acad Sci USA 1988 ; 85 : 2628-32.

40. Parmentier M, Libert F, Schurmans S, et al. Expression of members of the putative olfactory receptor gene family in mammalian germ cells. Nature $1992 ; 355$ : 453-5.

41. Zakeri Z, Welch W, Wolgemuth D. Characterization and inducibility of hsp 70 proteins in the male mouse germ line. J Cell Biol $1990 ; 111$ : 1785-92.

42. Allen R, O'Brien D, Jones C, Rockett D, Eddy E. Expression of heat shock proteins by isolated mouse spermatogenic cells. Moll Cell Biol 1988 ; 8 : 3260-6.

43. Höög C. Isolation of a large number of novel mammalian genes by a differential cDNA library screening strategy. Nucleic Acids Res $1991 ; 19$ : 6123-7.

44. Li H, Gyllensten U, Cui X, Saiki R Erlich H, Arnheim N. Amplification and analysis of DNA sequences in single human sperm and diploid cells. Nature 1988 ; 335 : 414. méiose [34]. Les gènes de ces isotypes spécifiques sont peu ou pas induits par la chaleur, ce qui pourrait expliquer la sensibilité des cellules germinales mâles à une élévation de la température. Cette absence d'inductibilité est cependant discutée, et la sensibilité à la chaleur pourrait aussi être due à la nature des protéines de choc thermique testiculaire $[41,42]$.

\section{Conclusion}

Une meilleure connaissance de l'expression des gènes au cours de la spermatogenèse pourrait trouver des applications pratiques et fondamentales. En effet, elle pourrait déboucher sur une meilleure compréhension de la physiopathologie de certaines stérilités mâles. A plus long terme, elle pourrait aussi conduire à de nouvelles approches de contraception masculine par des inhibiteurs spécifiques d'enzymes testiculaires ou par des procédés d'immunisation. Sur le plan fondamental, l'accumulation de données pourrait conduire à une meilleure connaissance des signaux de spécificité testiculaire, que ce soit au niveau des promoteurs, de l'épissage, de la polyadénylation, de la stabilité et de la traductibilité des messagers. Ce type d'information ne peut être obtenu que par une analyse systématique des messagers testiculaires. Cette analyse a déjà commencé chez la souris [43] et, dans notre laboratoire, chez l'homme. De plus, les nombreux isoenzymes ou isotypes présents dans le testicule constituent une réserve de mutations naturelles et pourraient aider aux études de structure-fonction des protéines. La technique d'amplification par PCR pourrait permettre de franchir encore une étape dans l'étude de l'expression des gènes testiculaires. En effet, il est maintenant possible d'amplifier une séquence d'ADN génomique à partir d'un seul spermatozoïde [44]. Cela permet d'analyser l'hétérogénéité des spermatozoïdes et d'effectuer des études de liaison génique à partir d'un seul donneur au lieu de plusieurs familles. Par une approche similaire, et si les progrès techniques permettent de réaliser la synthèse d'ADNc à partir d'une seule cellule, on pourra tester l'homogénéité de l'expression des gènes dans les spermatozoïdes

\section{Summary}

Gene expression during spermatogenesis

Male germ cells undergo a dramatic differentiation in testis leading to a highly specialized haploid cell, the spermatozoon. A specific pattern of gene expression corresponds to each stage of differentiation including post meiotic stages. This unique diversity in gene expression results from the use of a wide variety of regulatory mechanisms : genes that are specifically transcribed in germ cells, specific promoters, alternative splicing and polyadenylation. The physiological relevance of the expression of certain genes such as oncogenes, heat shock proteins genes, neuropeptides genes is still poorly understood. These questions are starting to be addressed now.

\section{TIRÉS A PART}

R. Barouki. 